OPEN ACCESS

Edited by:

Juan Sanchez-Esteban, Alpert Medical School of Brown

University, USA

Reviewed by:

MaryAnn Volpe,

Tufts University School of Medicine,

USA

Jill L. Maron,

Tufts Medical Center, USA

*Correspondence:

François Olivier

francois.olivier.1@ulaval.ca

Specialty section:

This article was submitted to Neonatology,

a section of the journal

Frontiers in Pediatrics

Received: 22 July 2016 Accepted: 12 September 2016 Published: 26 September 2016

Citation:

Olivier F, Nadeau S, Caouette G and Piedboeuf B (2016) Association between Apnea of Prematurity and

Respiratory Distress Syndrome in Late Preterm Infants: An Observational Study. Front. Pediatr. 4:105. doi: 10.3389/fped.2016.00105

\section{Association between Apnea of Prematurity and Respiratory Distress Syndrome in Late Preterm Infants: An Observational Study}

\author{
François Olivier*, Sophie Nadeau, Georges Caouette and Bruno Piedboeuf \\ Service of Neonatology, Department of Pediatrics, CHU de Québec-Université Laval, Quebec, QC, Canada
}

Background: Late preterm infants (34-36 weeks' gestation) remain a population at risk for apnea of prematurity (AOP). As infants affected by respiratory distress syndrome (RDS) have immature lungs, they might also have immature control of breathing. Our hypothesis is that an association exists between RDS and AOP in late preterm infants.

Objective: The primary objective of this study was to assess the association between RDS and AOP in late preterm infants. The secondary objective was to evaluate if an association exists between apparent RDS severity and AOP.

Methods: This retrospective observational study was realized in a tertiary care center between January 2009 and December 2011. Data from late preterm infants who presented an uncomplicated perinatal evolution, excepted for RDS, were reviewed. Information related to AOP and RDS was collected using the medical record. Odds ratios were calculated using a binary logistic regression adjusted for gestational age and sex.

Results: Among the 982 included infants, 85 (8.7\%) had an RDS diagnosis, 281 (28.6\%) had AOP diagnosis, and 107 (10.9\%) were treated with caffeine for AOP. There was a significant association between AOP treated with caffeine and RDS for all infants (OR $=3.3$, 95\% Cl: 2.0-5.7). There was no association between AOP and RDS in 34 weeks infants [AOR: 1.6 (95\% Cl: 0.7-3.8)], but an association remains for 35 [AOR: 5.7 (95\% Cl: 2.5-13.4)] and 36 [OR = 7.8 (95\% Cl: 3.2-19.4)] weeks infants. No association was found between apparent RDS severity and AOP, regarding mean oxygen administration duration or complications associated with RDS.

Conclusion: The association between RDS and AOP in late preterm infants reflects that patients affected by RDS are not only presenting lung immaturity but also respiratory control immaturity. Special consideration should be given before discontinuing monitoring after RDS resolution in those patients.

Keywords: apnea, respiratory distress syndrome

Abbreviations: AOP, apnea of prematurity; GA, gestational age; NICU, neonatal intensive care unit; RDS, respiratory distress syndrome. 


\section{INTRODUCTION}

Late preterm infants, born between $34^{0 / 7}$ and $36^{6 / 7}$ weeks gestational age (GA), account for an important proportion of newborns requiring neonatal intensive care, mainly because of respiratory morbidity (1-5). As an example, they have a significantly higher risk of respiratory distress syndrome (RDS) than term infants (RR: 17.3; 95\% CI, 9.8-30.6) (4). The incidence of RDS in late preterm infants was $15.4 \%$ in a study realized by the Canadian Neonatal Network (CNN) (6). Late preterm infants affected by RDS typically exhibit respiratory distress progressing over 48-72 h, associated with symptoms, such as tachypnea, nasal flaring, retraction, grunting, and hypoxemia. Apnea of prematurity $(\mathrm{AOP})$ is also a more frequent diagnosis in late preterm than in term infants, affecting up to $10 \%$ of those born at 34 and 35 weeks $(4,7,8)$. Since AOP is rarely objectified in the first few days of life, the diagnosis could easily be omitted with only a short period of monitoring $(9,10)$. Late preterm infants are also recognized to be at higher risk of unfavorable neurodevelopmental outcomes when compared with term infants (11-15). Developmental impacts of AOP are not well-known, and a causal relationship is not clearly established. However, some data raise great concerns about untreated AOP and neurodevelopmental impairments (16-19). As a consequence, pediatricians are daily confronted with monitoring issues and safe discharge concerning those infants. As AOP is a manifestation of immaturity, it might be worthwhile to pay attention to other immaturity manifestations, as they may be associated with AOP. This would help target more precisely which patient needs monitoring. We postulate that as late preterm infants affected by RDS have immature lungs, they may also have immature control of breathing and are at higher risk of AOP. Therefore, we hypothesized that RDS will be associated with AOP in late preterm infants. The primary objective of this study was to assess the association between RDS and AOP in a late preterm population. The secondary objective was to evaluate the association between apparent RDS severity and AOP in the population of late preterms affected by RDS.

\section{MATERIALS AND METHODS}

\section{Study Design and Population}

This is a tertiary care center, retrospective cohort study of the population of late preterm infants admitted between January 2009 and December 2011. Participants were identified through medical records review. The studied population included late preterm infants born or admitted to the Mother and Child Center of the CHU de Québec and who presented an uncomplicated perinatal evolution, except for RDS. We included only patients susceptible to be discharged soon after birth or after RDS resolution. The list of every diagnosis from the medical records was reviewed for each patient by the main author. Those who presented any medical or surgical condition that could significantly prolong the need for cardiorespiratory monitoring were excluded according to pre-specified criteria (Figure 1). The included patients were hospitalized either in the neonatal intensive care unit (NICU), in the newborn nursery, or in the moms' room. Frequent reasons for NICU admission at birth included respiratory distress and birth weight under $2.3 \mathrm{~kg}$ as per our protocol. All the patients admitted to the NICU received systematic cardiorespiratory monitoring. Those requiring intravenous infusion were hospitalized in the newborn nursery. This study was approved by the hospital ethics committee. Since this is a retrospective study and that all information collected were anonymous, no consent was required.

\section{Variables and Data Sources}

The primary outcomes of this study were AOP, and AOP treated with caffeine (caffeine citrate). Patients were reported having

- Congenital heart disease with hemodynamic or respiratory impact including patent ductus arteriosus requiring medical treatment with Ibuprofen

- Congenital disease of the airway and lungs or airway obstruction

- Chromosomal anomaly or genetic disease

- Congenital malformation

- Neuromuscular disorder including idiopathic or transient hypotonia of the newborn

- Early onset sepsis with positive blood, cerebrospinal fluid or urine culture

- Any condition that required surgery before discharge after birth

- Other medical conditions susceptible to prolong the hospitalization course (ex: pneumonia, PPHN, necrotizing enterocolitis, seizures, hypoxic ischemic encephalopathy, intra ventricular hemorrhage, multiple organ failure, hydrops, etc.)

- Death before discharge 
AOP and/or RDS diagnosis if these diagnoses were reported on the medical discharge summary. The diagnosis of AOP and RDS was confirmed on discharge summary by the attending physician who sent the patient home. To be classified as having a RDS, the patient must have required respiratory support and being hospitalized in the NICU. In the institution, AOP is defined by one of these three situations: the absence of breathing movements for $15 \mathrm{~s}$ or more, an abnormal respiratory pattern (bradypnea or superficial respiration) associated with oxygen saturation below $85 \%$, or bradycardia below 100 beats per minute (20). Distinction between central, obstructive, or mixed apnea was not possible. Caffeine treatment was documented according to the hospital's pharmacy database. Caffeine administration was based on clinical decision of the attending physician, according to frequency or severity of AOP, without pre-established criteria. GA and sex were considered as potential confounding factors for the main outcomes and were obtained from the medical records. Information from medical records and from pharmacy databases was merged according to chart numbers.

Secondary outcomes were only assessed in patients affected by RDS whether they presented AOP and/or received caffeine treatment or not. They included the duration of oxygen administration, ventilation support, and NICU hospitalization, as well as the combined outcome of mechanical ventilation exposure and pneumothorax. Ventilatory support included mechanical ventilation, non-invasive respiratory support/ventilation (nasal continuous positive pressure ventilation, nasal intermittent positive pressure ventilation, and sight positive airway pressure), and high flow nasal cannula. The charts of patients with RDS diagnosis have been reviewed to collect the data related to the secondary outcomes.

\section{Bias}

As AOP diagnosis were made clinically, without diagnostic evaluation for most of the patients, the outcome of AOP treated with caffeine was used as a proxy for infants with more significant AOP. This strategy was used in order not to overestimate the association between RDS and AOP.

\section{Statistical Methods}

Descriptive statistics are presented using percentages and means with SD. Adjusted analysis for sex and GA is presented using mean and SE. The primary outcomes were tested using a binary logistic regression model to obtain odds ratio. The simple effects between sex, GA, and RDS on AOP were assessed, and interaction was tested when necessary. Secondary outcomes were analyzed using an ANOVA, estimated with a linear mixed model for heterogeneous variances, to see the effects of caffeine or apnea, and adjusted for GA and sex. Bonferroni multiplicity correction was used when needed. All statistical analyses were performed with SAS, version 9.3 (SAS Institute Inc., Cary, NC, USA).

\section{RESULTS}

During the study period, 1101 late preterm infants were admitted to the Mother and Child Center of the CHU de Québec and $119(10.8 \%)$ were excluded according to the exclusion criteria
(Appendix A). Among all excluded patients, there were 35, 40, and 44 patients, respectively, aged from 34 to 36 weeks GA. Twenty (16.8\%) of them had a RDS diagnosis, and 37 (31.1\%) had an AOP diagnosis. Fifteen (12.6\%) had both AOP and RDS diagnosis.

Among the 982 patients forming the studied group, 85 (8.7\%) had a RDS diagnosis, 281 (28.6\%) had an AOP diagnosis, and 107 (10.9\%) were treated with caffeine for AOP. Patients with RDS diagnosis were all admitted to the NICU and required respiratory support. Among the 897 patients not affected by RDS, 65\% were admitted in the NICU.

Distribution according to GA and sex and descriptive data for the primary outcomes are presented in Table 1. The proportion of males increased from 34 to 36 weeks in the RDS group, but it remained stable in patients without RDS (Table 1). In the group of patients with RDS, 21 were exposed to mechanical ventilation and also received surfactant and 11 presented a pneumothorax.

\section{Primary Outcomes \\ Apnea of Prematurity}

There was a significant interaction between RDS and GA for the AOP outcome $(p=0.015)$. Therefore, AOP odds ratio were stratified by GA, and there was no more association between RDS and AOP in 34 weeks infants (OR = 1.6, 95\% CI: 0.7-3.7). Nonetheless, a significant association remains for $35(\mathrm{OR}=6.2$, 95\% CI: 2.6-14.6) and 36 (OR = 8.8, 95\% CI: 3.5-22.0) weeks infants. The overall AOP rate was $30.0 \%$ in females and $27.1 \%$ in males. Therefore, being a male was slightly associated with a decreased risk of AOP (OR $=0.69,95 \%$ CI: 0.49-0.97) according to the logistic regression model. There was no interaction between GA and sex $(p=0.33)$ neither between RDS and sex $(p=0.27)$ for the AOP outcome.

TABLE 1 | Study population.

\begin{tabular}{|c|c|c|}
\hline $\begin{array}{l}\text { Patients included in the study } \\
(N=982)\end{array}$ & $\begin{array}{l}\text { Patients with RDS } \\
\qquad(N=85)\end{array}$ & $\begin{array}{l}\text { Patients with no RDS } \\
(N=897)\end{array}$ \\
\hline Mean birth weight, grams (SD) & $2523(386)$ & 2504 (435) \\
\hline \multicolumn{3}{|l|}{ GA, $N(\%)$} \\
\hline $34^{0 / 7}$ to $36^{6 / 7}$ & 85 (100\%) & 897 (100\%) \\
\hline $34^{0 / 7}$ to $34^{6 / 7}$ & $35(41.1 \%)$ & $191(21.3 \%)$ \\
\hline $35^{0 / 7}$ to $35^{6 / 7}$ & $27(31.8 \%)$ & $244(27.2 \%)$ \\
\hline $36^{0 / 7}$ to $36^{6 / 7}$ & $23(27.1 \%)$ & $462(51.5 \%)$ \\
\hline
\end{tabular}

Males, $\boldsymbol{N}(\%)$

$34^{0 / 7}$ to $36^{6 / 7}$

$34^{0 / 7}$ to $34^{6 / 7}$

$35^{0 / 7}$ to $35^{6 / 7}$

$16 / 35(45.7 \%)$

$18 / 27(66.7 \%)$

$36^{0 / 7}$ to $36^{6 / 7}$

$18 / 23(78.3 \%)$

465/897 (51.9\%)

$106 / 191$ (55.2\%)

$123 / 244(50.6 \%)$

236/462 (51.2\%)

\section{AOP, $\boldsymbol{N}(\%)$}

$34^{0 / 7}$ to $36^{6 / 7}$

$34^{0 / 7}$ to $34^{6 / 7}$

$35^{0 / 7}$ to $35^{6 / 7}$

$36^{0 / 7}$ to $36^{6 / 7}$

$54 / 85$ (63.5\%)

$27 / 35(77.1 \%)$

$18 / 27(66.7 \%)$

$9 / 23(39.1 \%)$

227/897 (25.3\%)

$129 / 191(67.5 \%)$

$63 / 244(25.8 \%)$

$35 / 462(7.6 \%)$

$$
\begin{aligned}
& \text { Caffeine, } \boldsymbol{N}(\%) \\
& 34^{0 / 7} \text { to } 36^{6 / 7} \\
& 34^{0 / 7} \text { to } 34^{6 / 7} \\
& 35^{0 / 7} \text { to } 35^{6 / 7} \\
& 36^{0 / 7} \text { to } 36^{6 / 7}
\end{aligned}
$$




\section{AOP Treated with Caffeine}

Both GA $(\mathrm{OR}=3.13,95 \%$ CI 1.94-5.06, $p<0.0001)$ and RDS $(\mathrm{OR}=2.24,95 \%$ CI $1.26-3.97, p=0.0059)$ were individually associated with caffeine exposure according to the logistic regression model. There was no interaction between GA and RDS for that issue $(p=0.13)$. RDS was associated with AOP treated with caffeine $(\mathrm{OR}=3.3,95 \% \mathrm{CI}: 2.0-5.7)$. There was no interaction between GA and sex $(p=0.97)$ neither between RDS and sex $(p=0.66)$ for the outcome of being treated with caffeine for AOP.

\section{Secondary Outcomes}

Results of the secondary outcomes for the 85 patients affected by RDS are summarized in Table 2 and Table 3.

\section{Oxygen}

Mean oxygen duration for RDS was 2.3 (SD: 1.8) days for all patients. No significant relation was found between oxygen duration for RDS and AOP or caffeine administration for AOP.

\section{Ventilatory Support}

Mean ventilation support duration was 4.8 (SD: 2.8) days for all patients. Apnea diagnosis was associated with prolonged ventilation support, but this difference was not significant when addressing patients treated with caffeine.

\section{NICU Hospitalization}

Mean hospitalization duration in the NICU was 11.4 (SD: 7.1) days for all patients, and it was significantly longer in patients who presented AOP. The same association was observed for patients treated with caffeine, but the difference was borderline significant.

\section{RDS Complications}

No association was found in the regression model between $\mathrm{AOP}$ and the composite outcome of pneumothorax and/or

TABLE 2 | Secondary outcomes summary for patients with RDS regarding AOP.

\begin{tabular}{lcrc}
\hline $\begin{array}{l}\text { AOP, Yes } \\
\text { (54)/No (31) }\end{array}$ & $\begin{array}{c}\text { Unadjusted mean } \\
\text { (SD) }\end{array}$ & $\begin{array}{c}\text { Adjusted mean } \\
\text { (SE) }\end{array}$ & $\begin{array}{c}\text { ANOVA } \\
\text { p-value }\end{array}$ \\
\hline Oxygen duration (days) & $2.3(1.7) / 2.1(2.0)$ & $2.5(0.2) / 2.1(0.3)$ & 0.3 \\
Ventilation (days) & $5.4(3.0) / 3.7(1.9)$ & $5.2(0.3) / 4.0(0.4)$ & 0.02 \\
Hospitalization (days) & $12.5(8.2) / 8.4(3.3)$ & $12.3(1.1) / 8.9(0.7)$ & 0.01 \\
\hline
\end{tabular}

${ }^{a}$ No interaction where significant for GA and sex and therefore the model used does not consider it.

TABLE 3 | Secondary outcomes summary for patients with RDS regarding AOP treated with caffeine.

\begin{tabular}{lccc}
\hline $\begin{array}{l}\text { Caffeine for AOP, } \\
\text { Yes (22)/No (63) }\end{array}$ & Adjusted mean ${ }^{\text {(2E) }}$ & $\begin{array}{l}\text { ANOVA } \\
\boldsymbol{p} \text {-value }\end{array}$ & $\begin{array}{l}\text { ANOVA } \\
\boldsymbol{p} \text {-value }\end{array}$ \\
\hline Oxygen duration (days) & $1.5(1.4) / 2.5(1.9)$ & $1.8(0.4) / 2.6(0.2)$ & 0.08 \\
Ventilation (days) & $5.4(3.6) / 4.6(2.4)$ & $5.4(0.5) / 4.6(0.3)$ & 0.2 \\
Hospitalization (days) & $14.9(10.6) / 9.7(4.7)$ & $14.6(2.4) / 9.8(0.6)$ & 0.05 \\
\hline
\end{tabular}

${ }^{a}$ No interaction where significant for GA and sex and therefore the model used does not consider it. invasive ventilation exposure $(\mathrm{OR}=0.35,95 \% \mathrm{CI}: 0.12-1.03$, $p=0.06)$. This composite outcome was nonetheless associated with prolonged oxygen duration (3.1 vs. 2.1 days, $p=0.02$ ), but no association was found with ventilation support duration (4.6 vs. 5.1 days, $p=0.34$ ) neither hospitalization duration (10.8 vs. 11.6 days, $p=0.61$ ).

\section{DISCUSSION}

This study supports that late preterm infants suffering of RDS are at higher risk of AOP. Therefore, it confirms our research hypothesis regarding the association between respiratory manifestations of immaturity and AOP in late preterm infants. RDS was associated with AOP in 35 and 36 weeks infants, which are normally expected to show a higher level of respiratory control maturity, but not in 34 weeks infants. It is possible that patients of 34 weeks had a similar AOP rate in both groups because they have been more systematically monitored, even those without RDS. However, the association between AOP treated with caffeine and RDS was significant for all infants without interaction with GA. In fact, this association reflects the link between RDS and more significant AOP.

In 2011, Eichenwald et al. published a study addressing AOP very similar to our regarding inclusion criteria and data sources, but targeting 33 and 34 weeks' infants instead of late preterm (21). Therefore, the AOP incidence reported in their study (49\%) was superior to the one found in our study (29\%). Interestingly, they found a similar relation between AOP and length of hospital stay (21). In our study, there was no association either between duration of oxygen supplementation or RDS complications and the occurrence of AOP. This suggests that there is no relation between the severity of RDS and AOP. Nonetheless, we observed a significant interaction between duration of ventilation support and AOP in the RDS group. As there is no relation between oxygen supplementation and AOP, we hypothesized that the longer maintenance of ventilation support in that group was related to AOP rather than RDS.

Different mechanisms may contribute to the association between RDS and AOP in the population of late preterm infants. First, important changes in the dynamic of lung compliance occur until 38 weeks, contributing to the stabilization of the residual functional capacity $(1,22)$. The decreased in the residual functional capacity observed in RDS increase the susceptibility to more frequent and prolonged desaturations (1). Furthermore, RDS is thought to affect autonomic control in late preterm infants since it is associated with long-term decrease in heart rate variability, a pattern usually observed in more preterm infants. The mechanism explaining this autonomic variation needs to be better understand, but it may be related to RDS itself and could account for persistent $\operatorname{AOP}(8,23)$. Otherwise, it is known that late preterm infants have an immature respiratory response to hypoxia and to hyperoxia $(22,24)$. Dysregulation of peripheral chemoreceptors sensitivity, due to early postnatal hypoxemia, may be involved in the increase susceptibility to AOP in infants affected by RDS (24). Furthermore, chemoreceptors effectiveness remains dependent to the response of central mechanisms varying with the degree of brain maturation, which is not completed 
in late preterm infants (24). Additionally, the decline in ventilatory response associated with hyperoxia is known to increase the frequency of AOP, especially in those that exhibit periodic breathing, a frequent respiratory pattern present in late preterm infants $(22,25)$. This could have been an issue in patients exposed to prolonged oxygen administration for RDS, beyond the first days of life.

Clinicians' concerns about AOP are mainly related to hypoxic episodes and their impact on long-term neurological development. Although they are not clearly supported by the literature, potential life threatening events are also worrisome. It is known that late preterm infants are at higher risk of unfavorable neurodevelopmental outcomes when compared with term infants (11-15). Even though the causal relation between AOP and poor neurodevelopmental outcome is not clearly established, some data raise great concerns about this issue, mainly in extremely preterm infants $(16,17,19,26,27)$. Therefore, the optimal duration of cardiorespiratory monitoring in accordance with the natural evolution of AOP is unknown. Nonetheless, it is suggested that the first days of life are not the best window to proceed to a respiratory control diagnostic study (respirogram or pulse oximeter recording) (9). In our center, no infant is submitted to such an evaluation before 7 days of life and usually not before 39 weeks corrected age. Once an infant present AOP, he will remain on monitoring for up to 7 days after its last spell. Because cardiorespiratory monitoring is not mandatory, it may be discontinued shortly after RDS resolution if no AOP is documented during the initial monitoring. Evaluation is only requested for infants who present clinical manifestations of apnea, and no monitoring of the saturation in the car seat is performed in our institution before discharge as per new recommendations in Canada $(28,29)$.

Although our study included a large number of late preterm infants, we must acknowledge some of its limitations. On the one hand, the diagnosis of AOP was made clinically, and this may have underestimated the real incidence of AOP, mainly in 35 and 36 weeks infants without RDS. These patients were probably less likely to be monitored, while patients affected by RDS were systematically monitored. If this hypothesis is exact, it may have resulted in an increased likelihood of the association between $\mathrm{AOP}$ and RDS. On the other hand, we may have overestimated the global rate of AOP because of the diagnosis criteria used in our center. Indeed, AOP diagnostic criteria remained variable between institutions as the cut off for bradycardia may vary from 80 to 100 . Eichenwald et al. (21) reported this issue in their study pointing out the subjectivity of the diagnosis and the accuracy of data about AOP. They reported a rate of AOP diagnosis ranging

\section{REFERENCES}

1. Colin AA, McEvoy C, Castile RG. Respiratory morbidity and lung function in preterm infants of 32 to 36 weeks' gestational age. Pediatrics (2010) 126:115-28. doi:10.1542/peds.2009-1381

2. Engle WA, Tomashek KM, Wallman C, Committee on Fetus and Newborn, American Academy of Pediatrics. 'Late-Preterm' infants: a population at risk. Pediatrics (2007) 120:1390-401. doi:10.1542/peds.2007-2952

3. Kitsommart R, Janes M, Mahajan V, Rahman A, Seidlitz W, Wilson J, et al. Outcomes of late-preterm infants: a retrospective, single-center, Canadian study. Clin Pediatr (Phila) (2009) 48:844-50. doi:10.1177/0009922809 340432 from 24 to $76 \%$ among 10 sites. In our study, the 100 cut off point for bradycardia made the diagnosis more likely and therefore we may have overestimated the rates of AOP. Nonetheless, the same diagnosis criteria were used for all infants, and it should not have impacted the association between RDS and AOP. Furthermore, using the odds ratio for caffeine treatment allows to get a more significant picture from a clinical standpoint as patients treated with caffeine certainly presented more important manifestations of AOP either in severity or frequency. In rare cases, patients may have received caffeine before being discharged with home monitoring for periodic breathing, and this study did not allow us to identify those patients. However, periodic breathing is a pattern of alternating breaths and brief respiratory pauses and is considered as a marker of AOP (30).

\section{CONCLUSION}

Our study supports that RDS, a manifestation of pulmonary immaturity, is a marker for higher susceptibility of respiratory control immaturity in late preterm infants since it is associated with AOP. Therefore, special consideration should be given before discontinuing monitoring after RDS resolution. A prospective study addressing respiratory control would be an important step toward achieving clarity on the issue of AOP in late preterm infants. It could also allow to determine the best window and the appropriate duration for cardiorespiratory monitoring.

\section{AUTHOR CONTRIBUTIONS}

FO: wrote the manuscript, revised, and approved the final manuscript as submitted. SN: reviewed and revised the manuscript and approved the final manuscript as submitted. GC: reviewed and revised the manuscript and approved the final manuscript as submitted. BP: reviewed and revised the manuscript and approved the final manuscript as submitted.

\section{ACKNOWLEDGMENTS}

On behalf of the entire team, I would like to thank Anne-Sophie Julien, biostatistician at the Research Center of the CHU de Québec, for her essential contribution to this project.

\section{FUNDING}

This research received no specific grant from any funding agency in the public, commercial, or not-for-profit sectors.

4. Teune MJ, Bakhuizen S, Gyamfi Bannerman C, Opmeer BC, van Kaam AH, van Wassenaer AG, et al. A systematic review of severe morbidity in infants born late preterm. Am JObstet Gynecol (2011) 205:.e1-9. doi:10.1016/ j.ajog.2011.07.015

5. Consortium on Safe Labor, Hibbard JU, Wilkins I, Sun L, Gregory K, Haberman S, et al. Respiratory morbidity in late preterm births. JAMA (2010) 304:419-25. doi:10.1001/jama.2010.1015

6. Bassil KL, Shah PS, Shah V, Ye XY, Lee SK, Jefferies AL, et al. Impact of late preterm and early term infants on Canadian neonatal intensive care units. Am J Perinatol (2014) 31:269-78. doi:10.1055/s-0033-1347364

7. Zhao J, Gonzalez F, Mu D. Apnea of prematurity: from cause to treatment. Eur J Pediatr (2011) 170:1097-105. doi:10.1007/s00431-011-1409-6 
8. Hunt CE. Ontogeny of autonomic regulation in late preterm infants born at 34-37 weeks postmenstrual age. Semin Perinatol (2006) 30:73-6. doi:10.1053/ j.semperi.2006.02.005

9. Côté A, Hum C, Brouillette RT, Themens M. Frequency and timing of recurrent events in infants using home cardiorespiratory monitors. J Pediatr (1998) 132:783-9. doi:10.1016/S0022-3476(98)70304-X

10. Martin RJ, Wang K, Köroglu Ö, Di Fiore J, Kc P. Intermittent hypoxic episodes in preterm infants: do they matter? Neonatology (2011) 100:303-10. doi: $10.1159 / 000329922$

11. Chyi LJ, Lee HC, Hintz SR, Gould JB, Sutcliffe TL. School outcomes of late preterm infants: special needs and challenges for infants born at 32 to 36 weeks gestation. J Pediatr (2008) 153:25-31. doi:10.1016/j.jpeds.2008.01.027

12. Woythaler MA, McCormick MC, Smith VC. Late preterm infants have worse 24-month neurodevelopmental outcomes than term infants. Pediatrics (2011) 127:e622-9. doi:10.1542/peds.2009-3598

13. Quigley MA, Poulsen G, Boyle E, Wolke D, Field D, Alfirevic Z, et al. Early term and late preterm birth are associated with poorer school performance at age 5 years: a cohort study. Arch Dis Child Fetal Neonatal Ed (2012) 97:F167-73. doi:10.1136/archdischild-2011-300888

14. Talge NM, Holzman C, Wang J, Lucia V, Gardiner J, Breslau N. Late-preterm birth and its association with cognitive and socioemotional outcomes at 6 years of age. Pediatrics (2010) 126:1124-31. doi:10.1542/peds.2010-1536

15. McGowan JE, Alderdice FA, Holmes VA, Johnston L. Early childhood development of late-preterm infants: a systematic review. Pediatrics (2011) 127:1111-24. doi:10.1542/peds.2010-2257

16. Koons AH, Mojica N, Jadeja N, Ostfeld B, HiattM,Hegyi T. Neurodevelopmental outcome of infants with apnea of infancy. Am J Perinatol (1993) 10:208-11. doi:10.1055/s-2007-994719

17. Pillekamp F, Hermann C, Keller T, von Gontard A, Kribs A, Roth B. Factors influencing apnea and bradycardia of prematurity - implications for neurodevelopment. Neonatology (2007) 91:155-61. doi:10.1159/000097446

18. Corwin MJ, Hunt CE, Lister G, Tinsley LR, Baird T, Silvestri JM, et al. Cardiorespiratory events recorded on home monitors. JAMA (2001) 285(17):2199-207. doi:10.1001/jama.285.17.2199

19. Hunt CE, Corwin MJ, Lister G, Weese-Mayer DE, Ward SL, Tinsley LR, et al. Precursors of cardiorespiratory events in infants detected by home memory monitor. Pediatr Pulmonol (2008) 43:87-98. doi:10.1002/ppul.20745

20. Martin RJ, Abu-Shaweesh JM. Control of breathing and neonatal apnea. Biol Neonate (2005) 87:288-95. doi:10.1159/000084876

21. Eichenwald EC, Zupancic JA, Mao WY, Richardson DK, McCormick MC, Escobar GJ. Variation in diagnosis of apnea in moderately preterm infants predicts length of stay. Pediatrics (2011) 127:e53-8. doi:10.1542/ peds.2010-0495

22. Darnall RA, Ariagno RL, Kinney HC. The late preterm infant and the control of breathing, sleep, and brainstem development: a review. Clin Perinatol (2006) 33:883-914. doi:10.1016/j.clp.2006.10.004

23. Henslee JA, Schechtman VL, Lee MY, Harper RM. Developmental patterns of heart rate and variability in prematurely-born infants with apnea of prematurity. Early Hum Dev (1997) 47:35-50. doi:10.1016/S0378-3782(96)01767-7

24. Cardot V, Chardon K, Tourneux P, Micallef S, Stéphan E, Léké A, et al. Ventilatory response to a hyperoxic test is related to the frequency of short apneic episodes in late preterm neonates. Pediatr Res (2007) 62:591-6. doi:10.1203/PDR.0b013e318155868e

25. Abu-Shaweesh JM, Martin RJ. Neonatal apnea: what's new? Pediatr Pulmonol (2008) 43:937-44. doi:10.1002/ppul.20832

26. Hunt CE, Corwin MJ, Baird T, Tinsley LR, Palmer P, Ramanathan R, et al. Cardiorespiratory events detected by home memory monitoring and one-year neurodevelopmental outcome. J Pediatr (2004) 145(4):465-71. doi:10.1016/ j.jpeds.2004.05.045

27. Baird TM. Clinical correlates, natural history and outcome of neonatal apnoea. Semin Neonatol (2004) 9:205-11. doi:10.1016/j.siny.2003.11.007

28. Whyte RK, Hilliard RI, Jefferies AL, Peliowski-Davidovich A, Sorokan ST, Whyte HEA. Safe discharge of the late preterm infant. Paediatr Child Health (2010) 15(10):655-60.

29. Narvey MR, Society CP, Committee N. Assessment of cardiorespiratory stability using the infant car seat challenge before discharge in preterm infants $(<37$ weeks' gestational age). Paediatr Child Health (2016) 21(3):155-62.

30. Mathew OP. Apnea of prematurity: pathogenesis and management strategies. J Perinatol (2011) 31:302-10. doi:10.1038/jp.2010.126

Conflict of Interest Statement: The responsible author, FO, has no disclosure. The other authors have no conflicts of interest to disclose. The authors declare that the research was conducted in the absence of any commercial or financial relationships that could be construed as a potential conflict of interest.

Copyright (c) 2016 Olivier, Nadeau, Caouette and Piedboeuf. This is an open-access article distributed under the terms of the Creative Commons Attribution License (CC $B Y)$. The use, distribution or reproduction in other forums is permitted, provided the original author(s) or licensor are credited and that the original publication in this journal is cited, in accordance with accepted academic practice. No use, distribution or reproduction is permitted which does not comply with these terms. 


\section{APPENDIX}

\section{A. 119 Excluded patients per categories}

1. Congenital heart disease with hemodynamic or respiratory impact including patent ductus arteriosus requiring medical treatment with Ibuprofen

$$
\text { - } 7 \text { patients }
$$

2. Congenital disease of the airway and lungs and airway obstruction

- 6 patients

3. Chromosomal anomaly and genetic disease

- 11 patients

4. Congenital malformation

- 26 patients
5. Neuromuscular disorder including idiopathic or transient hypotonia of the newborn

$$
\text { - } 6 \text { patients }
$$

6. Early onset sepsis with positive blood, cerebrospinal fluid, or urine culture

- 7 patients

7. Any condition that required surgery before discharge after birth

- 16 patients

8. Other medical conditions susceptible to prolong the hospitalization course (e.g., pneumonia, PPHN, necrotizing enterocolitis, seizures, hypoxic ischemic encephalopathy, IVH, multiple organ failure, and hydrops)

- 39 patients

9. Death before discharge

- 1 patient 